\title{
Para una hermenéutica de la enemistad: los estudios culturales latinoamericanos y el nuevo fascismo
}

Towards a Hermeneutics of Enmity: Latin American

Cultural Studies and the New Fascism

Para uma hermenéutica da inimizade: os estudos

culturais latino-americanos e o novo fascismo

\section{Jens Andermann}

UNIVERSITÄT ZÜRICH， SUIZA

Profesor del Departamento de Español y Portugués de la Universidad de Zurich. PhD en Estudios Latinoamericanos por la Universidad Libre de Berlín. Por más de diez años ha integrado el Consejo Editorial del fournal of Latin American Cultural Studies.

Entre sus publicaciones se destacan los libros New Argentine Cinema / Nuevo cine argentino (I.B. Tauris, 2011 / Paidós, 2015), The Optic of the State / A Óptica do Estado (University of Pittsburgh Press, 2007 / Editora UERJ, 2014) y Mapas de poder: una arqueología literaria del espacio argentino (Beatriz Viterbo Editora, 2000), así como los libros editados La escena y la pantalla: cine contemporáneo y el retorno de lo real (Colihue, 2014; con Álvaro Fernández Bravo), New Argentine and Brazilian Cinema: Reality Effects (Palgrave, 2013; con Álvaro Fernández Bravo), Galerías del progreso: museos, exposiciones y cultura visual en América Latina (Beatriz Viterbo Editora, 2006; con Beatriz González-Stephan) e Images of Power: Iconography, Culture and the State in Latin America (Berghahn Books, 2003; con William Rowe). Correo electrónico: anderjens@gmail.com 
Ensayo

Documento accesible en línea desde la siguiente dirección: http://revistas.javeriana.edu.co

Traducción de Rodrigo del Río Joglar. Estudiante doctoral, Department of Romance

Languages \& Literatures, Harvard University. Correo electrónico: rdelriog@harvard.edu

doi:10.11144/Javeriana.c121-41.heec

RECIBIDO: 11 DE ENERO DE 2016. ACEPTADO: 22 DE FEBRERO DE 2016. DISPONIBLE EN LÍNEA: 26 DE JUNIO DE 2017

\section{Cómo citar este ensayo:}

Andermann, Jens. "Para una hermenéutica de la enemistad: los estudios culturales latinoamericanos y el nuevo fascismo". Cuadernos de Literatura 21.41 (2017): 79-89. https://doi.org/10.11144/Javeriana.cl21-41.heec 
No PARECE GASUAL que el término 'emergencia' detente, en español y portugués, un tamaño potencial crítico, especialmente ahora que nos enfrentamos, en toda América Latina, una vez más, con formas catastróficas de cambio. El momento histórico que atestiguamos, a mi parecer, más que un mero colapso de los desarrollismos neokeynesianos impulsados por la caída en los precios de las commodities o una restauración del neoliberalismo al estilo de los noventa, asiste a la emergencia de regímenes militantemente reaccionarios, basados en novedosas articulaciones de un consumismo resentido, provocado incansablemente por oligopolios comunicacionales con agendas transnacionales y geopolíticas vinculadas al capitalismo financiero. Un nuevo bloque de poder de antigua raigambre ha reunido a jugadores que, hasta ahora, solían alternar entre equipos rivales, con el fin de desplazar de las riendas del Estado a sus contenedores de la "marea rosa". El capital rentista, basado en la posesión de tierras, ha unido fuerzas con el capital especulativo, tal como lo han hecho las viejas y nuevas clases letradas: la corporación jurídica y los conglomerados comunicacionales, que en conjunto producen la música ambiental de los nuevos regímenes en clave de pospolítica y pánico moral las 24 horas del día. Por falta de un mejor término, podríamos hablar de un fascismo del shopping, respaldado con frecuencia por un evangelismo maniqueo, que imagina la salvación, no en forma de un Valhala nacional como sus predecesores modernistas, sino más bien como un Miami universal, incluidos sus bronceados playeros, cuentas offshore y elecciones con papeletas de voto perdidas. En el nuevo-viejo nombre del progreso, de la eficiencia, de la transparencia y la posideología, un ethos cosmopolita (onda Google, Twitter, iPhone, antes que Internacional de los Trabajadores) ha sido reclutado al servicio de una redistribución simbólica y material desinhibida y brutal. La reducción vertiginosa del acceso a bienes primarios y derechos ciudadanos hacia la que actualmente se encaminan Argentina y Brasil, para no mencionar a Paraguay, Guatemala u Honduras, parece señalar una intención, no solamente de revertir, sino incluso de erradicar de una buena vez las mismas condiciones de posibilidad de las agencialidades que posibilitaron algunas difíciles victorias en las áreas de la justicia social y los derechos humanos en los tardíos noventa y comienzos del 2000.

Nada de esto será noticia para quien siga estos eventos a través de otros medios que no sean los del mainstream corporativo. Lo que me asombra, no obstante, es el grado en el que los estudios culturales latinoamericanos, el campo en el que muchos revestimos nuestra práctica intelectual y académica cotidiana, han fallado en siquiera comenzar a abordar este salto a la derecha que, sostengo, se fundamenta en una cultura de la guerra de ya larga data. 
Como sabe cualquiera que haya visitado recientemente Brasil o Argentina, la polarización entre coxinhas y petiscos, o entre $K$ y anti- $K$, ha alcanzado ahí niveles de animosidad que exceden, de lejos, cualquier instancia anterior en las últimas décadas. ¿Cómo pudo una disciplina cuyos objetivos, para citar la útil definición de Abril Trigo, son "la producción simbólica y las experiencias vivas de la realidad social latinoamericana ... como estrechamente conectadas a relaciones sociales, políticas y materiales" (Trigo 3-4), haber fracasado tan calamitosamente al abordar la reconfiguración del campo de "producción simbólica' que se ha puesto en curso al menos desde el ascenso al poder de Hugo Chávez en 1998 y que, con las recientes derrotas electorales del Chavismo y del Kichnerismo, o con los golpes jurídico-parlamentarios en Honduras, Paraguay y Brasil, parece haber llegado al fin de su trayecto, para ser sucedida por un total asalto político, jurídico, mediático y, donde fuera necesario, militar a cualquier forma de poder popular organizado? Que luchemos hoy por siquiera nombrar el monstruo que nos enfrenta nos indica también una de las razones de este fracaso: por casi dos décadas, los estudios culturales latinoamericanos han descuidado su quehacer principal de - para citar nuevamente a Trigo- indagar en la producción recíproca de lo cultural y "de lo que es experimentado en las esferas social y política" (Trigo 4). Desfasaje, sugiero, que se debe a que los propios vocabularios críticos del campo han permanecido inmunes a los lenguajes apasionados de lo político forjados en Latinoamérica sobre el final del milenio. $\mathrm{O}$, más grave aun, los lenguajes de los estudios culturales latinoamericanos, surgidos en el marco de los 'nuevos movimientos sociales' y las políticas identitarias, ahí donde no se han convertido en instancias anémicas y puramente auto-referenciales de un izquierdismo de campus, han hecho poco para resistir su cooptación activa por un 'Republicanismo' espurio que denuncia la arremetida populista contra la 'democracia' o por la versión de la nueva derecha - tamizada por las relaciones públicas - de los eslóganes de tolerancia, participación, sustentabilidad, y así sucesivamente.

El quincuagésimo aniversario de LASA en 2016, que coincide con el vigesimoquinto volumen del fournal of Latin American Cultural Studies, es una oportunidad propicia para reflexionar sobre esta debacle. Para hacerlo, sin embargo, debemos parar de contarnos la historia familiar de nuestro campo como si fuese una historia de éxitos y más bien mirar hacia atrás, como el ángel de Benjamin, a través de un campo de escombros, al mismo tiempo que de usar en nuestra propia ventaja el momento de peligro en el que nos encontramos, como el haz de luz que ilumina y nos permite reconocer los fragmentos y piezas que puedan vertirse, aún, contra un enemigo que apenas conocemos porque, 
en tanto armas, comienzan a definir los órganos y funciones del cuerpo en contra del que son blandidas. Esta es, en resumidas cuentas, la apuesta de la 'antología' que los editores del fournal estamos preparando actualmente para celebrar el vigésimo quinto aniversario de la revista: asumir tanto el fracaso $y$ el potencial aún vigente del proyecto de estudios culturales latinoamericanos, escudriñando en los archivos una vez más en busca de proposiciones excéntricas, off-mainstream, cuya productividad solo hoy somos capaces de reconocer cuando se desenvuelven sus sentidos preñados de futuro.

Fundada inicialmente como Traves/sia, el objetivo manifiesto de la revista había sido abrir un espacio de traducción y de mutuo enriquecimiento entre varios campos todavía en estado de 'formación', para usar el término de Raymond Williams (Marxism and Literature 124), incluyendo los variados modos en los que el concepto de 'cultura' estaba entonces reconfigurando constelaciones disciplinares en la academia británica y estadounidense, pero también dando lugar, en Latinoamérica, a conversaciones inéditas sobre medios audiovisuales desarrollados entre tradiciones literarias y antropológicas junto con estudios sociales y comunicacionales, todo en el contexto de una reconsideración post-1989 de la política y la estrategia de la izquierda. Al mismo tiempo, en su doble grafía, referenciada por una ensombrecida segunda 's', Traves/sia también sostuvo su alianza con un radicalismo estético que muchos de estos nuevos 'culturalismos' estaban ahora viendo con sospecha; uno que la revista subsecuentemente reafirmó a través de colaboraciones de la talla de Néstor Perlongher, Margo Glantz, Raúl Zurita o João Gilberto Noll. 'Travesía', en español, había sido también un concepto clave del experimento poéticoartístico de Amereida, realizado por un colectivo de arquitectos, escritores y artistas de Valparaíso que partirían a fundar la 'Ciudad Abierta' en 1972: una reclamación lírica así como performativa del espacio histórico-geográfico, que el portugués 'Travessia' pone en una intricada y, de algún modo, enigmática relación con el monólogo épico Grande Sertão: Veredas de João Guimarães Rosa, del que es la última palabra y oración final; y no menos porque tanto el término español como el portugués están también íntimamente conectados, en su semántica histórica, con el viaje y el descubrimiento y, por tanto, con la colonialidad del conocimiento.

En un acto similar de balance que, digamos, el de la Revista de Crítica Cultural organiza en Chile dada su cercana relación con los legados estéticos de la 'escena de avanzada', la revista buscó reconciliar una poética radical con una hermenéutica de la cultura - para parafrasear el título de un libro de William Rowe (1996), uno de sus fundadores- y así también traducir no 
únicamente hacia y desde diferentes locaciones geopolíticas y lingüísticas de la 'teoría cultural', sino también entre esta y una producción estética neo- o postvanguardista que, como el crítico de arte Mário Pedrosa había anticipado ya en 1966, era en sí misma "ya no puramente artística sino más bien cultural" (Pedrosa 207) en su alcance y materialidades. Como en cualquier traducción, una considerable cantidad de ruido se desencadenó en este intento de mediar entre idiomas cuyos propósitos e inclinaciones frecuentemente tiraban en direcciones opuestas, como se vuelve obvio al releer hoy el primer par de volúmenes de la revista, con sus "cuestionarios de estudios culturales" impresos al lado de trabajos ensayísticos y narrativo-experimentales, con frecuencia bruscamente "cambiando entre ... la crítica cultural latinoamericana y el latinoamericanismo crítico de los Estados Unidos", como lo formuló John Kraniauskas (3) en un temprano balance de la revista más de diez años atrás, añadiendo: "aquí yace nuestro drama (el de la revista)". Este drama, sugiero, consistía no menos en tratar de proveer un espacio discursivo para intercambios entre varios desvíos críticos del modo de crítica ideológica de la Escuela de Frankfurt o del Althusserianismo - los estudios culturales inspirados en la escuela de Birmingham, las nuevas teorías de medios y mediaciones surgidas en torno de FLACSO, así como más tarde posiciones subalternistas y poscoloniales- al mismo tiempo que la revista se aferraba a un ethos de negatividad estética, o al menos a la 'excepcionalidad' del hecho estético, sin reactivar por eso figuraciones profético-trágicas de los artistas/intelectuales como los que cultivaban modos de crítica anteriores al giro cultural.

Esta 'primera ola' culturalista sería sometida, ella misma, a incisivas críticas en la revista por parte de autores como Ricardo Kaliman en 1998, Ana del Sarto en 2000, o Gabriela Nouzeilles en 2001, entre otros, quienes aludían precisamente al costo intelectual y político que los estudios culturales estaban pagando por su divorcio de corrientes anteriores, en gran parte de inspiración marxista, de reflexionar críticamente sobre la cultura. Estas, desde Mariátegui hasta Retamar, habían concebido a la cultura como un campo de batalla ideológica en donde el crítico estaba interviniendo, en palabras de Arturo Jauretche, "colocándome 'a la paleta" en el método" (9); esto es, como "un actor y observador apasionado" en vez de asumir el punto de vista imparcial del académico transnacional.

Irónicamente - o no- las críticas de Kaliman, Del Sarto y Nouzeilles estaban apareciendo en el momento en que la revista, y el 'campo' al que esta ahora proveía, estaba experimentando un rápido proceso de institucionalización. Esta se manifestaba, por ejemplo, en el paso del inicial modo de producción y distribución artesanal al editor académico Carfax (subsecuentemente Taylor 
\& Francis), que coincidió con la promoción del anterior subtítulo al nombre bajo el que la revista ha estado circulando desde entonces, cortando aun más sus amarres — señalados por el nombre Traves/sia - en genealogías regionales estéticas y críticas. El mutable colectivo de intelectuales que editaba la revista - provenientes de la academia anglo-americana, pero también de Latinoamérica y Europa - estaba consciente de las aporías que implicaba este acto de malabarismo. Optamos así por explicitar el desfasaje entre el ambiente intelectual latinoamericano y los protocolos profesionales de la economía del conocimiento estadounidense y británica a través de una intensificada 'política de la traducción". Nuevas secciones tales como "Desde el archivo" permitieron por primera vez a audiencias anglófonas acceder al trabajo de Ángel Rama y Antonio Cornejo Polar, o a las incursiones sudamericanas del Collège de Sociologie, entre otros, mientras que la revista también documentó y tradujo controversias al interior de Latinoamérica tales como el debate en torno a la carta abierta de Oscar del Barco, No Matarás, en 2006-2007. "Dispatches" (Despachos), una nueva rúbrica introducida desde el 2010 para breves crónicas ensayísticas escritas 'sobre el filo' del acontecer de eventos y procesos políticos y sociales, busca reavivar algo del espíritu del testimonio intelectual invocado por Jauretche. Hasta ahora, las contribuciones han cubierto las muertes de Néstor Kirchner y Hugo Chávez; el discurso anti-terrorista bajo la segunda presidencia de Alan García y la alimentación forzada de prisioneros mapuches en Chile; los protestos previos a la Copa del Mundo en Brasil, y el movimiento estudiantil mexicano contra el fraude electoral, incluyendo también dossiers especiales sobre la elección del primer Papa latinoamericano y el ascenso del macrismo en Argentina.

Sin embargo, los 'datos de usuario' que nos facilitan en intervalos regulares nuestros distribuidores comerciales, revelan una preferencia - no sorprendente, por otra parte- por una serie más consolidada de tópicos, índice, acaso, de los significados y el rol de los estudios culturales para las aulas de clases de hoy en día. De un tiempo a ahora, el artículo 'mejor vendido' de la revista ha sido una pieza sobre la importancia de la cultura dominicana y centroamericana de lucha libre en la novela Brief and Woundrous Life of Oscar Wao de Junot Díaz — una novela escrita en inglés (Garland Mahler)—. Con toda probabilidad, el aporte 'latinoamericanista' es buscado aquí como 'trasfondo cultural' desde fuera del campo mismo, mientras que otros tópicos populares con los lectores - cine mexicano, brasileño y argentino contemporáneo, en particular filmes ambientados en márgenes urbanas, la obra de Roberto Bolaño, o la memoria postdictatorial en el Cono Sur-son al menos indicativos de los contenidos actuales de los planes de estudio de pregrado 
en programas de Español y Portugués en el Reino Unido y los Estados Unidos. En comparación, los propios intentos de la revista por fomentar agendas diferentes y reconectar con la política off-campus, tal como se ha estado desarrollando a lo largo de Latinoamérica misma desde la perspectiva del análisis cultural, han conocido un éxito bastante más limitado, especialmente ahí donde no han circulado en forma de acceso libre. De hecho, la comodificación del trabajo crítico y su circulación a través de plataformas conectadas a una economía del conocimiento en gran parte privatizada - en donde los autores pueden escoger hoy entre canales 'gold' y 'platinum' de acceso en línea para sus escritos- son proclives a obstruir el encuentro de audiencias heterodoxas que la revista buscaba facilitar en sus comienzos. El fenómeno es aun más grave en Latinoamérica misma en donde, por razones lingüísticas pero también debido al acceso vastamente desigual a paquetes comerciales de bibliotecas, la revista casi no circula en absoluto, con la excepción del neoliberalizado sistema universitario chileno y de un puñado de universidades privadas en otros países: no precisamente la audiencia que se tenía en mente al momento de la fundación de Traves/sia. Intentos por abordar el problema a través de versiones de libre acceso en español y portugués de la revista en la plataforma en línea de Taylor \& Francis han fracasado consistentemente: los repositorios de saber anglo-americano no atienden a tales regionalismos fuera del mercado principal de su interés.

No se trata, por supuesto, de lamentar la vida difícil del editor de revistas académicas, sino de reflexionar sobre la medida en que la condición de posibilidad de nuestra producción crítica se encuentra inmersa hoy en lógicas tecno-económicas que son quizás indiferentes antes que hostiles a modos particulares de pensamiento y que, sin embargo, complican la posibilidad de mantener el tipo de conversaciones a dos o tres bandas de las que el campo de estudios culturales latinoamericanos emergió y del que extrajo su raison d'être ética y política. El uso de plataformas multilingües en línea, auspiciadas por universidades públicas o privadas, tales como las que han introducido revistas en línea como A Contracorriente y alter/nativas, podría proveer una solución aunque sea parcial para ese problema. Sin embargo, me parece que, hasta ahora, ninguna de esas iniciativas ha dado con la Piedra de la Sabiduría, menos aun cuando se trata de trascender el autismo político que caracteriza el estado contemporáneo de nuestro campo. Con ello vuelvo a mis observaciones iniciales sobre nuestra incapacidad -no necesariamente en tanto individuos, pero ciertamente dentro de las prácticas y terminologías de las disciplinas que profesamos- para responder al nuevo fascismo en la cultura política de 
Latinoamérica. Debemos reflexionar sobre los modos en que las economías políticas del conocimiento, en cuyo dominio institucional nuestros propios esfuerzos están inexorablemente atrapados, forcluyen hoy en día determinados modos de trabajo sustentados por figuras y gestos de negatividad intelectual y estética - un proceso que Willy Thayer puntillosamente asoció, en una conversación acontecida más de diez años atrás, con el concepto político, económico y administrativo de "transición" (Thayer et al.)—. Aun más, estas mismas economías parecen favorecer activamente una cierta pragmática del 'análisis cultural' que, en un cierto momento, podría haber ofrecido una salida de determinados callejones sin salida de la crítica ideológica. No obstante, hoy en día su complicidad - activa o pasiva - con los procesos de la subsunción de la vida (del que la 'economización' del pensamiento es una parte y parcela) se ha vuelto poco menos que innegable.

El 'acceso libre' no es una llave mágica, en la medida en que continúa sirviendo a la lógica de los 'índices de impacto' y resultados cuantificables, lo que inevitablemente debe hacer al interior de una academia mercantilizada. Más bien, me parece, la pregunta hoy es sobre cómo, desde el interior de nuestras prácticas profesionales inescapablemente inmersas en sistemas de auto-reificación y neoliberalización del pensamiento, podemos no obstante recobrar algo de la 'hermenéutica de la enemistad' que todavía movilizaba a corrientes de crítica cultural en Latinoamérica que los estudios culturales pugnaron por superar. Con esto me refiero al ejercicio paradójico, frecuentemente llevado a cabo bajo condiciones de peligro y adversidad extrema, de forjar intuiciones en torno al universo afectivo y simbólico de un 'otro' que, a pesar de la sensibilidad y casi amorosa atención al detalle en el análisis de sus hábitos y modos de expresión, nunca cesó de ser identificado como 'enemigo'. Este modo de lectura, que productivamente alternó "la sospecha y el goce", para citar el título de la genealogía de la crítica cultural en Chile escrita por Ana del Sarto y publicada en 2010, de hecho también había estado en el origen mismo de las aproximaciones modernas a la 'cultura', como Michel de Certeau famosamente mostró en su genealogía de 1974 de los "estudios del folkore" en la Francia posrevolucionaria. Sin embargo, recibió su giro más productivo al ser aplicado a la cultura de masas del siglo XX producida industrialmente, desde el "ornamento de la masa" de Kracauer a las críticas de Debord y Marcuse a las culturas del espectáculo del capitalismo tardío; por los que podríamos fácilmente substituir una genealogía latinoamericana paralela, digamos, desde las reflexiones de Mariátegui sobre Chaplin, a la crítica de Dorfman y Mattelart a Disney, vía la sociología histórica de El medio pelo en la sociedad argentina 
(1966) de Jauretche, del que extraía mis citas anteriores. Sospecha y goce, o quizás debiésemos decir una capacidad para la compenetración sensitiva -Einfühlung en alemán- y un agudo, incluso apasionado, antagonismo hacia sus sujetos, juntos producían la tensión productiva desde la que esta hermenéutica de la enemistad forjó sus intuiciones - una forma de leer diametralmente opuesta, sugiero, a la hermenéutica de la empatía que ha predominado en los estudios culturales latinoamericanos-, culminando quizás en los debates alrededor del testimonio en la mitad de los noventa. Por supuesto, las formas y modos de experiencia histórica que estas modalidades de interpretación antagonística apuntan a acceder —alienación versus autenticidad, por así decir - fueron y continúan siendo de un orden muy diferente, y no estoy sugiriendo que el péndulo debiese simplemente balancearse hacia atrás en dirección de una crítica de la ideología de la vieja escuela. Sin embargo, si hubo alguna cosa a la que los estudios de testimonio podrían haber prestado más atención, en lugar de enfocar todas sus energías en la mística de la voz y la presencia, era precisamente al agudo conocimiento que estas voces transmitieron sobre quién era su enemigo y de lo que era capaz: algo, sostengo, que los estudios culturales urgentemente necesitan reaprender hoy.

\section{Obras citadas}

Del Sarto, Ana . "Cultural Critique in Latin America or Latin American Cultural Studies?". Fournal of Latin American Cultural Studies 9.3 (2000): 235-247. Impreso.

Del Sarto, Ana. Sospecha y goce: una genealogía de la crítica cultural en Chile. Santiago de Chile: Cuarto Propio, 2010. Impreso.

De Certeau, Michel. "La beauté du mort". La culture au pluriel. Paris: Seuil, 1974. 45-72. Impreso.

Dorfman, Ariel y Armand Mattelart. Para leer al Pato Donald. Comunicación de masa y colonialismo. México, D. F.: Siglo XXI, 1972. Impreso.

Garland Mahler, Anne. "The Writer as Superhero: Fighting the Colonial Curse in Junot Diaz's The Brief and Wondrous Life of Oscar Wao". Fournal of Latin American Cultural Studies 19.2 (2010): 119-140. Impreso.

Jauretche, Arturo. El medio pelo en la sociedad argentina. Apuntes para una sociología nacional. Buenos Aires: Peña Lillo, 1966. Impreso.

Kaliman, Ricardo. "What is 'Interesting' in Latin American Cultural Studies". Fournal of Latin American Cultural Studies 7.2 (1998): 261-272. Impreso.

Kraniauskas, John. "The Cultural Turn? On the Journal of Latin American Cultural Studies (1992-2004)". Revista de Estudios Hispánicos 39.3 (2005): 561-569. Impreso. 
Nouzeilles, Gabriela. "Apocalyptic Visions: National Tales and Cultural Analysis in a Global Argentina". Fournal of Latin American Cultural Studies 10.3 (2001): 291-301. Impreso.

Pedrosa, Mário. "Arte ambiental, arte pós-moderna, Hélio Oiticica". Correio da Manhã 26 (June 1966). Publicado nuevamente en: Dos murais de Portinari aos espaços de Brasília. São Paulo: Perspectiva, 1981. 205-209. Impreso.

Rowe, William. Hacia una poética radical. Ensayos de hermenéutica cultural. México, D. F.: Fondo de Cultura Económica, 2014. Impreso.

Thayer, Willy, Guadelupe Santa Cruz, Federico Galende y Pablo Oyarzún. "Conversation on Willy Thayer's The Unmodern Crisis of the Modern University". Nepantla: Views From South 1.1 (2000): 229-254. Impreso.

Trigo, Abril. "General Introduction". The Latin American Cultural Studies Reader. Ed. Ana del Sarto, Alicia Ríos y Abril Trigo. Durham: Duke University Press, 2004. 1-14. Impreso.

Williams, Raymond. Marxism and Literature. Oxford: Oxford University Press, 1977. Impreso. 